\title{
Controlling plasmonic resonances in binary metallic nanostructures
}

\author{
Ying Gu, ${ }^{1, a)}$ Jia Li, ${ }^{1}$ Olivier J. F. Martin, ${ }^{2}$ and Qihuang Gong ${ }^{1, b)}$ \\ ${ }^{1}$ Department of Physics, State Key Laboratory for Mesoscopic Physics, Peking University, Beijing 100871, \\ China \\ ${ }^{2}$ Nanophotonics and Metrology Laboratory, Swiss Federal Institute of Technology Lausanne (EPFL), \\ EPFL-STI-NAM, ELG Station 11, CH-1015 Lausanne, Switzerland
}

(Received 8 February 2010; accepted 24 March 2010; published online 8 June 2010)

\begin{abstract}
Investigation on the interplay of plasmonic resonances in binary nanostructures indicated that, at a fixed wavelength, with a variation in the difference permittivity ratio $\eta=\left(\epsilon_{2}-\epsilon_{0} / \epsilon_{1}-\epsilon_{0}\right)$, resonances exhibit the dielectric effect, resonance chaos, collective resonance, resonance flat, and new branch regions. This means that plasmonic resonances can be controlled by material parameters $\epsilon_{1}$ and $\epsilon_{2}$. In this work, using the Green's matrix method of solving the surface plasmon resonances, we first study the resonance combination of symmetrical binary three-nanostrip systems. Several resonance branches extend across the above mentioned regions. Near fields within the gaps and at the ends of nanostrips are greatly enhanced due to the influence of neighboring metallic material. Then, along each resonance branch, resonances in the dielectric permittivity region are mapped into the wavelength region of gold. Through adjusting material parameters $\epsilon_{1}$ and $\epsilon_{2}$, the resonance wavelength is tuned from $\lambda_{R}=500$ to $1500 \mathrm{~nm}$, while for a single nanostrip it is only at $\lambda_{R}$ $=630 \mathrm{~nm}$. We also find that comparable permittivity parameters $\epsilon_{1}\left(\right.$ or $\left.\epsilon_{2}\right)$ and $\epsilon_{\mathrm{Au}}(\omega)$ can control resonance wavelength and intensity effectively. High dielectric permittivity of the neighboring metal has also an advantage in a giant enhancement of the near field. These findings provide new insights into design of hybrid plasmonic devices as plasmonic sensors. (C) 2010 American Institute of Physics. [doi:10.1063/1.3407527]
\end{abstract}

\section{INTRODUCTION}

Surface plasmon resonances (SPRs) of metallic nanoparticles enable important applications based on surfaceenhanced Raman scattering, ${ }^{1}$ biosensors and nanometer plasmonic waveguides, ${ }^{2}$ optical antennas, ${ }^{3}$ solar cells, ${ }^{4,5}$ and nonlinear optical frequency mixing. ${ }^{6-8}$ In practice, it is necessary to adjust the resonance wavelength as well as to design the enhanced near field. There are several ways of approaching this problem. One is to fabricate different types of nanoparticles such as nanospheres, nanoshells, nanorices, nanorings, nanostars, nanocages, and nanotriangles. ${ }^{2}$ By controlling the core-shell ratios of nanoshells, resonances can be tuned from visible to infrared frequencies. ${ }^{2}$ The nanocrescents have shown multiple, adjustable resonances and a strongly enhanced near field due to their unique structures with tips and a ring. ${ }^{9}$ Another way is to arrange the nanoparticles in different configurations and to use the near field coupling among them to tailor their plasmonic properties. For example, the redshifts and blueshifts of resonance wavelength and giant near field enhancement in nanoparticle pairs have been reported, ${ }^{10-14}$ as well as the scaling rules of shifts. ${ }^{15,16}$ Other than using the geometry and arrangement of nanostructures, here we propose utilizing their material parameters to control their plasmonic properties. This is especially effective when both kinds of nanostructures are metal-

\footnotetext{
a)Electronic mail: ygu@pku.edu.cn.

${ }^{b)}$ Electronic mail: qhgong@pku.edu.cn.
}

lic, their plasmonic properties are greatly modified by the presence of the free electrons of the neighboring metal.

Based on the Green's tensor method to solve the optical near field of isolated nanostructures and the Green's function formalism in the quasistatic limit, ${ }^{17,18}$ we have developed the Green's matrix method (GMM) to deal with the SPRs and the near field of arbitrary shape subwavelength metallic structures and of binary nanostructures. ${ }^{19,20}$ Instead of giving the resonance peaks in the wavelength space, by using the resonance capacity of surface plasmons, ${ }^{19}$ the GMM can provide the resonance spectrum in the dielectric permittivity space. By varying the difference permittivity ratio $\eta=\left(\epsilon_{2}\right.$ $\left.-\epsilon_{0}\right) /\left(\epsilon_{1}-\epsilon_{0}\right)$, at a fixed wavelength, resonance branches of two nanostrips exhibit different regions: the dielectric effect region, resonance chaos region, collective resonance region, resonance flat region, and new branches region. ${ }^{20}$ This means that the plasmonic resonance wavelength and the near field distribution can be adjusted by means of the material parameters of nanostructures. It is well known that a symmetrical structure has an incomparable advantage in plasmonic properties. In the present work, by means of the developed method, binary triple-nanostrip systems with symmetrical arrangements are utilized to control the SPR properties. These three-strip-based plasmonic building blocks can be used to design various tunable hybrid plasmonic devices such as plasmonic sensors.

In the following section, the GMM and resonance capacity concept are briefly reviewed. In Sec. III, we present the resonance combination of binary three-nanostrips at the fixed 
(a)

$\varepsilon_{1} \quad \varepsilon_{2} \quad \varepsilon_{1}$

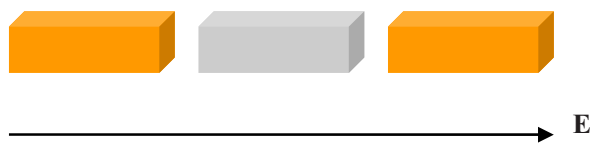

(b)

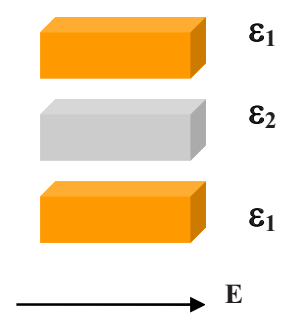

FIG. 1. (Color online) Scheme of three $60 \times 20 \times 20 \mathrm{~nm}^{3}$ nanostrips for (a) parallel case and (b) perpendicular case. Here the $x$ axis is the direction of polarization of the incident light. The propagation is along the $z$ axis.

wavelengths $\lambda=632.8$ and $832 \mathrm{~nm}$. It is shown that (i) several resonance branches, which have different resonance capacities and permittivity parameter ranges, extend across the above mentioned regions and (ii) near fields concentrated within the gaps or at the ends of the nanostrips are greatly enhanced by the free-electrons in the neighboring metallic materials. The characteristics of each branch can act as a guide for using real metal parameters to control the plasmonic properties. Then, in Sec. IV, we describe the mapping of the resonance properties in the dielectric permittivity region into the wavelength region of the real metal gold along these resonance branches. By adjusting the material parameter $\epsilon_{1}$ (or $\epsilon_{2}$ ), the resonance wavelength of the system can be tuned from $\lambda_{R}=500$ to $1500 \mathrm{~nm}$, compared to its single nanostrip resonance at $\lambda_{R}=630 \mathrm{~nm}$. Simultaneously, the near field is strongly enhanced. This control of the resonance wavelength requires that the permittivity parameter $\epsilon_{1}$ (or $\epsilon_{2}$ ) is comparable with $\epsilon_{\mathrm{Au}}(\omega)$, while high dielectric permittivity of the neighboring metal can lead to significient enhancement of the near fields. Finally, we summarize the main results in Sec. V.

\section{GMM}

The salient properties of the GMM are first reviewed. ${ }^{19,20}$ Consider an arbitrary shape binary structure with dielectric permittivities $\epsilon_{1}(\mathbf{r}, \omega)$ and $\epsilon_{2}(\mathbf{r}, \omega)$ embedded in a homogeneous bulk material $\epsilon_{0}(\omega)$, as shown in Fig. 1 . These nanostructures are not necessarily connected, but the tensors $\epsilon_{\mathrm{Si}}(\mathbf{r}, \omega)\left[=\epsilon_{i}(\mathbf{r}, \omega)-\epsilon_{0}(\omega)\right.$ for $i=1$ or 2] vanish outside the structures. Dielectric permittivities $\epsilon_{1}(\mathbf{r}, \omega)$ and $\epsilon_{2}(\mathbf{r}, \omega)$ are generally complex and frequency dependent. If a monochromatic field $\mathbf{E}^{0}(\mathbf{r}) e^{-i \omega t}$ impinges on the system, the scattered field $\mathbf{E}(\mathbf{r})$ is a solution of the wave equation

$$
-\nabla \times \nabla \times \mathbf{E}(\mathbf{r})+k^{2} \epsilon_{0}(\omega) \mathbf{E}(\mathbf{r})+k^{2} \epsilon_{\mathrm{Si}}(\mathbf{r}, \omega) \mathbf{E}(\mathbf{r})=0,
$$

where $k$ is the vacuum wave number. By introducing the Green's operator $\mathbf{G}^{0}\left(\mathbf{r}, \mathbf{r}^{\prime}, \omega\right)$ associated with bulk material, ${ }^{21}$ the Lippmann-Schwinger equation of the field $\mathbf{E}(\mathbf{r})$ at any point $\mathbf{r}$ can be written as ${ }^{17,22}$

$$
\begin{aligned}
\mathbf{E}(\mathbf{r})= & \mathbf{E}^{0}(\mathbf{r})+k^{2} \int_{c_{1}} d \mathbf{r}^{\prime} \mathbf{G}^{0}\left(\mathbf{r}, \mathbf{r}^{\prime}, \omega\right) \boldsymbol{\epsilon}_{s 1}\left(\mathbf{r}^{\prime}, \omega\right) \cdot \mathbf{E}\left(\mathbf{r}^{\prime}\right) \\
& +\eta k^{2} \int_{c_{2}} d \mathbf{r}^{\prime} \mathbf{G}^{0}\left(\mathbf{r}, \mathbf{r}^{\prime}, \omega\right) \boldsymbol{\epsilon}_{s 1}\left(\mathbf{r}^{\prime}, \omega\right) \cdot \mathbf{E}\left(\mathbf{r}^{\prime}\right),
\end{aligned}
$$

where $C=C_{1} \cup C_{2}$ denotes the cluster subspace, and $\eta=\left(\epsilon_{2}\right.$ $\left.-\epsilon_{0}\right) /\left(\epsilon_{1}-\epsilon_{0}\right)$ is the difference permittivity ratio. ${ }^{20}$ This equation implies that the field inside the nanoclusters can be solved self-consistently through the Green's propagator $\mathbf{G}^{0}\left(\mathbf{r}, \mathbf{r}^{\prime}, \omega\right)$.

If the nanocluster subspace is discretized into $N$ pieces with volume $\delta V \quad(\delta V \ll V)$, let the Green's matrix $\widetilde{\mathbf{G}^{0}}\left(\mathbf{r}, \mathbf{r}^{\prime}, \omega\right)=\delta V k^{2} \mathbf{G}^{0}\left(\mathbf{r}, \mathbf{r}^{\prime}, \omega\right) \delta\left(\mathbf{r}^{\prime}-\mathbf{r}_{1}\right)$

$+\eta \delta V k^{2} \mathbf{G}^{0}\left(\mathbf{r}, \mathbf{r}^{\prime}, \omega\right) \delta\left(\mathbf{r}^{\prime}-\mathbf{r}_{2}\right)$ for $\mathbf{r}_{1} \in C_{1}$ and $\mathbf{r}_{2} \in C_{2}$. Equation (2) becomes

$$
\sum_{\mathbf{r}^{\prime} \in C}\left[\widetilde{\mathbf{G}^{0}}\left(\mathbf{r}, \mathbf{r}^{\prime}, \omega\right)-s \mathbf{I}\right] \cdot \widetilde{\mathbf{E}}(\mathbf{r})=\frac{-\widetilde{\mathbf{E}^{0}}(\mathbf{r})}{\epsilon_{s 1}(\mathbf{r}, \omega)},
$$

where $s=\left(1 / \epsilon_{s 1}(\mathbf{r}, \omega)\right)=\left(1 / \epsilon_{1}(\mathbf{r}, \omega)-\epsilon_{0}(\omega)\right)$ is the eigenvalue of $\widetilde{\mathbf{G}^{0}}\left(\mathbf{r}, \mathbf{r}^{\prime}, \omega\right)$. Because $\widetilde{\mathbf{G}^{0}}$ is symmetrical, mathematically, there are $3 N$ real eigenvalues. Physically, only those eigenvalues with large residues of the electric field correspond to strong resonances, which are selected by the resonance capacity of the surface plasmon. ${ }^{19}$ The region of eigenvalue $s_{n}$ is clearly related to the ratio $\eta$. The cooperation of positive permittivity (here $\epsilon_{0}=1.0$ ) and negative permittivity $\epsilon_{1 n}(r, \omega)$ or $\epsilon_{2 n}(r, \omega)$ leads to the resonances.

Via some transformations, the field $\widetilde{\mathbf{E}}(\mathbf{r})($ a $3 N \times 1$ matrix) within the cluster subspace has the form

$$
\widetilde{\mathbf{E}}(\mathbf{r})=\sum_{n=1}^{3 N} \frac{\mathbf{L}_{n} \cdot \widetilde{\mathbf{E}^{0}}(\mathbf{r})}{\epsilon_{s 1}(\mathbf{r}, \omega)\left(s-s_{n}\right)} \mathbf{R}_{n}
$$

with eigenvalues $s_{n}$ and eigenvectors $\mathbf{R}_{n}$ and $\mathbf{L}_{n}$ of the Green's matrix $\widetilde{\mathbf{G}^{0}}$. For any point $\mathbf{r}$ outside the nanoclusters, $E(\mathbf{r})$ reads

$$
\begin{aligned}
\mathbf{E}(\mathbf{r})= & \mathbf{E}^{0}(\mathbf{r})+k^{2} \sum_{n=1}^{3 N} \frac{\mathbf{L}_{n} \cdot \widetilde{\mathbf{E}^{0}}(\mathbf{r})}{\left(s-s_{n}\right)}\left\{\left[\sum_{\mathbf{r}^{\prime} \in C_{1}} \mathbf{G}^{0}\left(\mathbf{r}, \mathbf{r}^{\prime}, \omega\right)\right.\right. \\
& \left.\left.+\eta \sum_{\mathbf{r}^{\prime} \in C_{2}} \mathbf{G}^{0}\left(\mathbf{r}, \mathbf{r}^{\prime}, \omega\right)\right] \cdot \mathbf{R}_{n}\right\} .
\end{aligned}
$$

For any binary nanostructure with $\epsilon_{1}(\mathbf{r}, \omega)$ and $\epsilon_{2}(\mathbf{r}, \omega)$, both the optical near field and far field can be expressed by the Green's tensor $\mathbf{G}^{0}\left(\mathbf{r}, \mathbf{r}^{\prime}, \omega\right)$.

When $s$ approaches one of the eigenvalues $s_{n}$, i.e., $s$ $\rightarrow s_{n}$, the electric fields diverge to infinity when there is no loss. Only those eigenstates (or resonances) with strong near electric fields are physically meaningful. ${ }^{1-8}$ To select those SPRs, we have defined the resonance capacity in the function of the internal energy of the nanostructures. ${ }^{19}$ In binary nanostructures, for each $s_{n}$, the joint resonance capacity is 


$$
C_{n}=\frac{\int_{C_{1}} d \mathbf{r}^{\prime}\left|\epsilon_{1 n}\right| \cdot\left[\operatorname{res}\left|\mathbf{E}\left(\mathbf{r}^{\prime}\right)\right|\right]_{n}^{2}+\int_{C_{2}} d \mathbf{r}^{\prime}\left|\epsilon_{2 n}\right| \cdot\left[\operatorname{res}\left|\mathbf{E}\left(\mathbf{r}^{\prime}\right)\right|\right]_{n}^{2}}{\int_{C} d \mathbf{r}^{\prime}\left|\epsilon_{0}(\omega)\right| \cdot\left|\mathbf{E}^{0}\left(\mathbf{r}^{\prime}\right)\right|^{2}},
$$

where the residue of field $\left[\operatorname{res}\left|\mathbf{E}\left(\mathbf{r}^{\prime}\right)\right|\right]_{n}$ $=\left|\mathbf{L}_{n} \cdot \mathbf{E}^{0}\left(\mathbf{r}^{\prime}\right) / \epsilon_{1 n}(\mathbf{r}, \omega) \mathbf{R}_{n}\right|$. Large resonance capacity values usually represent strong resonances with a strongly enhanced near field. Conceptually, extinction peaks correspond to those SPRs with high values of the resonance capacity. In the three-nanostrip case, instead of using the resonance capacity of each nanostrip as in the two-nanostrip case, ${ }^{20}$ we use the joint resonance capacity to represent the resonance intensity.

\section{SPR AND NEAR FIELD DISTRIBUTIONS}

The $\eta-s$ diagram is used to describe the SPR distribution with varying $\eta$ at a fixed wavelength, as well as the $\eta$ $-C_{n}$ (resonance capacity) diagram to give information on the near field enhancement. Through investigations in the $\eta-s$ and $\eta-C_{n}$ diagrams for binary nanostrips, it is found that resonance branches include the following regions: the dielectric effect region, resonance chaos region, collective resonance region, resonance flat region, and new branch region, ${ }^{20}$ which means that the plasmonic resonance properties can be adjusted by $\eta$, especially when both kinds of nanostructures are metallic, i.e., $\eta>0$. The main task of this section is to present the $\eta-s$ and $\eta-C_{n}$ diagrams of symmetrical threestrip nanostructures at $\lambda=632.8$ and $832 \mathrm{~nm}$. Since each branch has a different resonance capacity and dielectric permittivity range, in practice, we can roughly determine the type of real metal with suitable plasmonic properties if we know which branch it belongs to. Then, it is easy to map resonance branches in the permittivity region to the wavelength region. Based on the results presented here, in Sec. III A, we will demonstrate how to use the material parameters to control plasmonic properties in a wide spectral region.

As shown in Fig. 1(a), when the central line of the threenanostrips is parallel to the polarization of the external electric field, it is a parallel case, however, in Fig. 1(b), when their central line is perpendicular to the polarization of the electric field, it is a perpendicular case. Here the input wavelength is $\lambda=632.8$ or $832 \mathrm{~nm}$, the size of a nanostrip is 60 $\times 20 \times 20 \mathrm{~nm}^{3}$, and all the gaps are $10 \mathrm{~nm}$. In the calculations, we use a mesh of $5 \mathrm{~nm}$. The electric field is propagating along the $z$ direction and polarized along the $x$ direction. In the calculations of near field distributions, we select the $x y$ plane within the metal and $10 \mathrm{~nm}$ above the nanostrips with the imaginary parts $0.15 i$ in the parallel case and $0.05 i$ in the perpendicular case. When the incident wavelength is $\lambda$ $=632.8 \mathrm{~nm}$, for a single $60 \times 20 \times 20 \mathrm{~nm}^{3}$ nanostrip, there is only one resonance at $\epsilon_{1}(r, \omega) \approx-11.78$, roughly corresponding to gold material at this wavelength. ${ }^{19}$

\section{A. Parallel case}

Figure 2 displays the evolution of the resonance branches, resonance capacity, and relative dielectric permittivities $\epsilon_{1}$ and $\epsilon_{2}$ as a function of the difference permittivity ratio $\eta$ at $\lambda=632.8 \mathrm{~nm}$ for the parallel case in Fig. 1(a). Note that here only resonances with dielectric permittivities within the visible spectral region are considered. With the help of the $\eta-C_{n}$ diagram, we can select several main resonance branches. Roughly speaking, a binary three-strip nanostructure exhibits resonance behavior similar to a binary two-strip nanostructure. ${ }^{20}$ However, resonance branches and the details which lay the foundation of Sec. IV are quite different. When $\eta<0$, it is a dielectric effect region, i.e., there must exist one dielectric material among $\epsilon_{1}$ and $\epsilon_{2}$. As shown in Fig. 2(a), when $\epsilon_{1}$ is metallic, it is branch 1 but when $\epsilon_{2}$ is metallic, it is branch 2. For $\eta>0$, both materials are metallic. When 0 $<\eta<1$, branches 3 and 4 appear, where branch 3 belongs to the resonance chaos region because there is a large fluctuation of the resonance capacity, as shown in Fig. 2(b). Branches 4 and 7 belong to the new branch region, where only one kind of metallic material dominates the resonance behavior, i.e., in branch $4, \epsilon_{2}$ dominates, while in branch 7, $\epsilon_{1}$ dominates. Near $\eta=1$ with $\epsilon_{1}=\epsilon_{2}$, branches 5 and 6 are within the collective resonance region where both materials participate in the resonances, nevertheless, beyond $\eta>1.5$, branch 6 enters the resonance flat region with a quite strong plasmonic resonance.
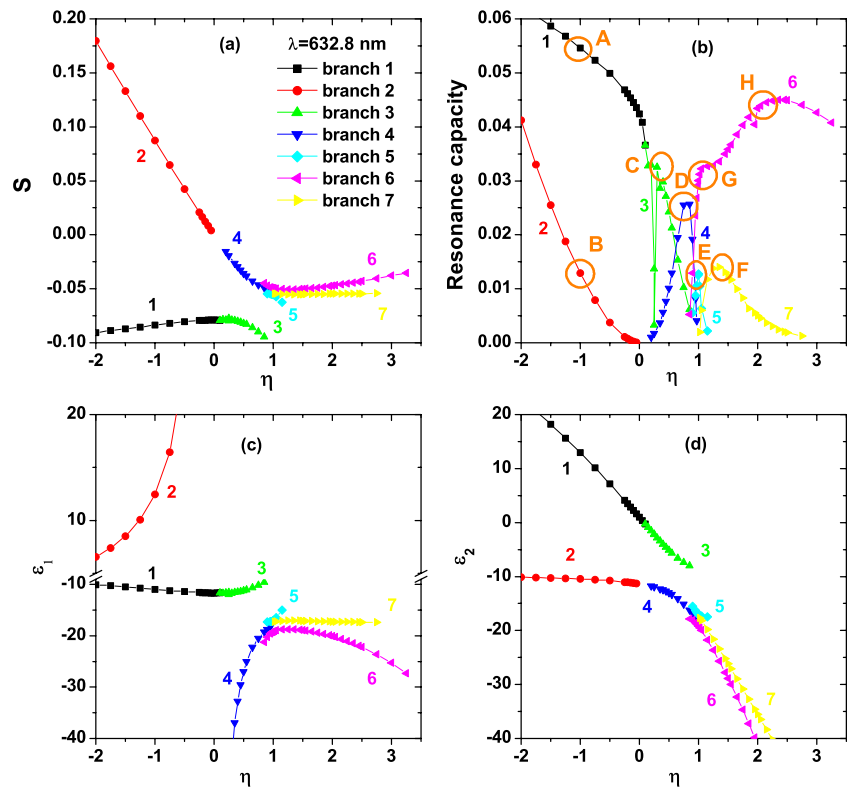

FIG. 2. (Color online) SPR with varying $\eta$ for the parallel case shown in Fig. 1(a) and at the wavelength $\lambda=632.8 \mathrm{~nm}$. (a) $\eta-s$ diagram, (b) $\eta-C_{n}$ diagram, (c) eigendielectric permittivity $\epsilon_{1}$, and (d) eigendielectric permittivity $\epsilon_{2}$. There are seven branches whose dielectric permittivities fall into the optical frequency range. 

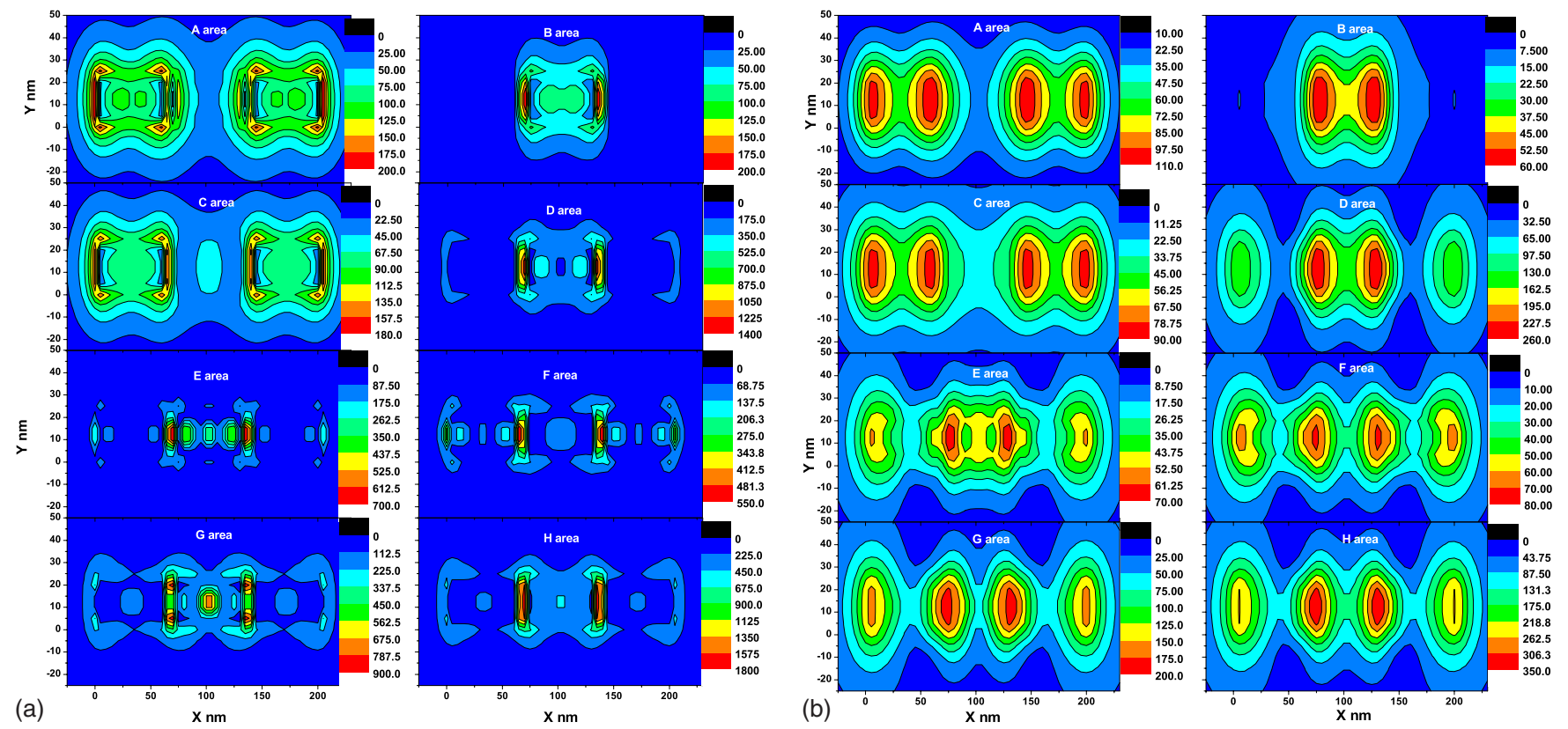

FIG. 3. (Color online) Near field distributions (a) within the nanostrips and (b) $10 \mathrm{~nm}$ above the nanostrips around the areas $A, B, C, D, E, F, G$, and $H$ marked in Fig. 2(b).

Detailed resonance behavior in branches 1 and 2 are first discussed. In the dielectric effect region, the existence of the neighboring dielectric nanostrip has some influence on the resonance capacity as shown in Fig. 2(b). Because electromagnetic waves can penetrate through the dielectric material, its resonance behavior is the same as in the vacuum, namely, the resonance energy is gathered within or around the metallic material. When $\eta=-1$, two resonances belonging to branches 1 and 2 are found. One is dominated by $\epsilon_{1}$ material with $\epsilon_{1}=-10.94$ and $\epsilon_{2}=12.94$ and the other is dominated by $\epsilon_{2}$ with $\epsilon_{1}=12.46$ and $\epsilon_{2}=-10.46$, see Figs. 2(c) and 2(d). Their near field in areas $A$ and $B$ is shown in Figs. 3(a) and 3(b). In these two branches, when the dielectric constants of the dielectric material greatly increase, the near field is only slightly enhanced and the dielectric permittivities of the metals are very stable. This implies that, although the high dielectric constant of the neighboring dielectric is used, it cannot be used to adjust the resonance wavelength and the near field enhancement effectively.

Now we start to explore branches 3,4 , and 7 shown in Fig. 2. They have a common feature in that only one metal material dominates the resonance while the other metal has a large effect on it. Branch 3 is very special due to the alternation of $\epsilon_{1}$ and $\epsilon_{2}$ dominating resonances. In this branch, the near fields around area $C$ are illustrated in Fig. 3, which is a resonance dominated by $\epsilon_{1}$ with $\epsilon_{1}=-11.70$ and $\epsilon_{2}=-2.80$. Though the maximum intensity of the near field is comparable with the $A$ area in branch 1 , different from where there is no field enhancement in the dielectric material $\epsilon_{2}$, there are still large near fields in the $\epsilon_{2}$ material. In branch 4 , resonances are dominated by $\epsilon_{2}$, and it is seen from the $D$ area with $\epsilon_{1}=-22.26$ and $\epsilon_{2}=-14.12$ in Fig. 3 that the near fields of the three-nanostrips are inseparable. While, in branch 7 , the $\epsilon_{1}$ material dominates the resonances, which are typically illustrated by the $F$ area with $\epsilon_{1}=-17.08$ and $\epsilon_{2}=-23.40$ in Fig. 3. In these branches, from the parameter ranges of $\epsilon_{1}$ and $\epsilon_{2}$ in Figs. 2(c) and 2(d), we see that $\epsilon_{2}$ has a large varying region in branches 3 and 7 and $\epsilon_{1}$ has a large varying region in branch 4 , which can be used to adjust the resonance wavelength of real metals. However, in branches 4 and 7 , there is a giant near field enhancement within the gaps, which is caused by the redistribution or polarization of electrons ${ }^{23}$ in the neighboring metal and can be applied to various plasmonic devices. ${ }^{1-5}$

Next, let us focus on branches 5 and 6 in Fig. 2. When $\eta=1$ and $\epsilon_{1}=\epsilon_{2}$ and there exist two collective resonances shown as area $G$ and area $E$ of Fig. 3. When $\epsilon_{1}=\epsilon_{2}=$ -17.06 , the resonance belongs to branch 5 , when $\epsilon_{1}=\epsilon_{2}=$ -19.20 , it belongs to branch 6 . The characteristic of collective resonances is that all three-nanostrips participate in the resonances, which leads to a large near field enhancement in the gaps. ${ }^{10-13}$ With an increment of $\eta$, the collective resonance in branch 6 becomes a $\epsilon_{1}$ dominated resonance. When $\eta=2, \epsilon_{1}=-20.12$, and $\epsilon_{2}=-41.25$, and the near field intensity reaches the maximum, which is nine times that of a single nanostrip due to the existence of free-electrons in neighboring metallic nanostructure $\epsilon_{2}$. Hence, a large $\epsilon_{2}$ in branch 6 can be used to control the resonance wavelength and resonance intensity when these resonances are mapped into the wavelength region of a real metal.

\section{B. Perpendicular case}

We consider the resonance properties for the perpendicular case of a three-nanostrip system, as shown in Fig. 1(b). In this situation, the near field coupling among them is not as strong as in the parallel case. There are 5 main resonance branches in the optical frequencies. In the $\eta-s$ and $\eta-C_{n}$ (resonance capacity) diagrams of Fig. 4, we see that branches 1 and 2 belong to the dielectric effect region, branch 3 roughly spans the resonance chaos region, collective resonance region, and resonance flat region, and branches 4 and 5 

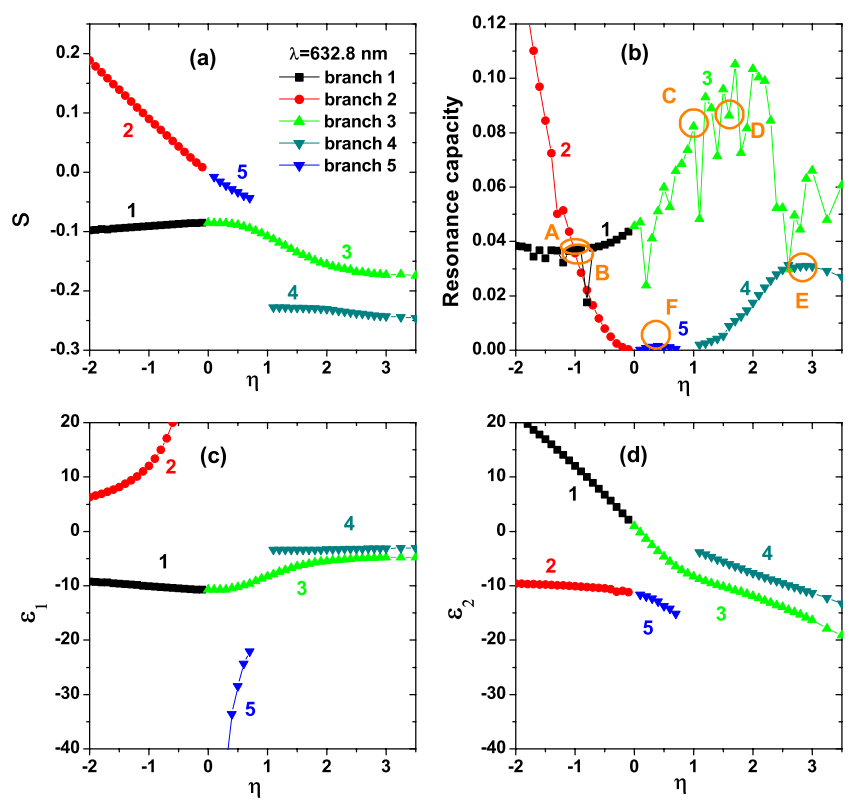

FIG. 4. (Color online) SPR with varying $\eta$ for the perpendicular case shown in Fig. 1(b) and at the wavelength $\lambda=632.8 \mathrm{~nm}$. (a) $\eta-s$ diagram, (b) $\eta$ $-C_{n}$ diagram, (c) eigendielectric permittivity $\epsilon_{1}$, and (d) eigendielectric permittivity $\epsilon_{2}$. There are five branches whose dielectric permittivities fall into the optical frequency range.

belong to the new branch region. The three-strip binary nanosystem takes on resonance behaviors similar to the twostrip case, ${ }^{20}$ yet the details are quite different.

First consider the two dielectric branches 1 and 2 with $\eta<0$. Since the electromagnetic wave can penetrate though

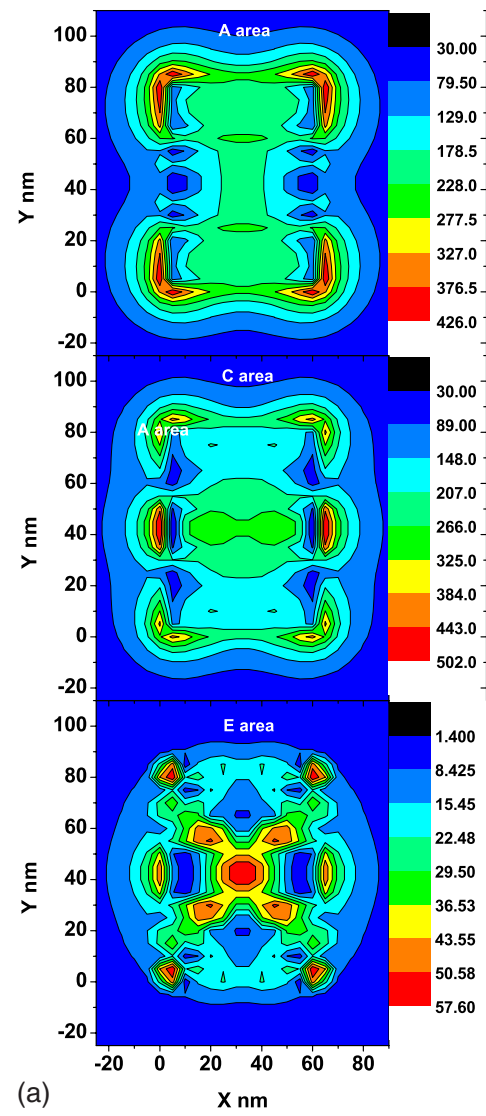

the dielectric material, varying $\eta$ does not influence the resonance capacity much, as shown in Fig. 4(b). When $\eta=-1$, two resonances are found. One is $\epsilon_{1}$ material dominated resonance with $\epsilon_{1}=-10.06$ and $\epsilon_{2}=12.06$ and the other is $\epsilon_{2}$ dominated with $\epsilon_{1}=10.07$ and $\epsilon_{2}=-12.07$, see Figs. 4(c) and 4(d). Their near fields are concentrated near the metals, as shown in the $A$ and $B$ areas in Fig. 5. Changing the dielectric constants of the dielectric did not have a large effect on the near field or the resonant dielectric permittivities of the metal, which means that the dielectric materials cannot be utilized to adjust the resonance properties.

For branch 3, due to the coexistence of multibranches, there exist fluctuations of the resonance capacity in branch 3 . $^{20}$ Here only the strongest resonances are selected, so not every individual branch is clearly shown. As an example, we give here the details for the collective resonance. When $\eta$ $=1.0$ with $\epsilon_{1}=\epsilon_{2}=-8.27$, Fig. 5 at the $C$ area shows a strong near field coupling at the ends of the nanostrips. When $\eta$ $=1.6$ with $\epsilon_{1}=-6.19$ and $\epsilon_{2}=-10.50$, area $D$ with enhanced near field distribution in Fig. 5 is the extension of the collective region and is transformed to a resonance flat region. In this branch, a large range of $\epsilon_{2}$ and resonance capacity implies an effective control of the resonances in the wavelength region of real metals.

Branches 4 and 5 belong to the new branch region. In branch 4 , resonances are dominated by $\epsilon_{1}$ material. When $\eta=2.8$ with $\epsilon_{1}=3.15$ and $\epsilon_{2}=-10.61$, the plot of the $E$ area in Fig. 5 represents a typical near field distribution in branch 4. It is seen that the intensity of the near field is not high, yet a

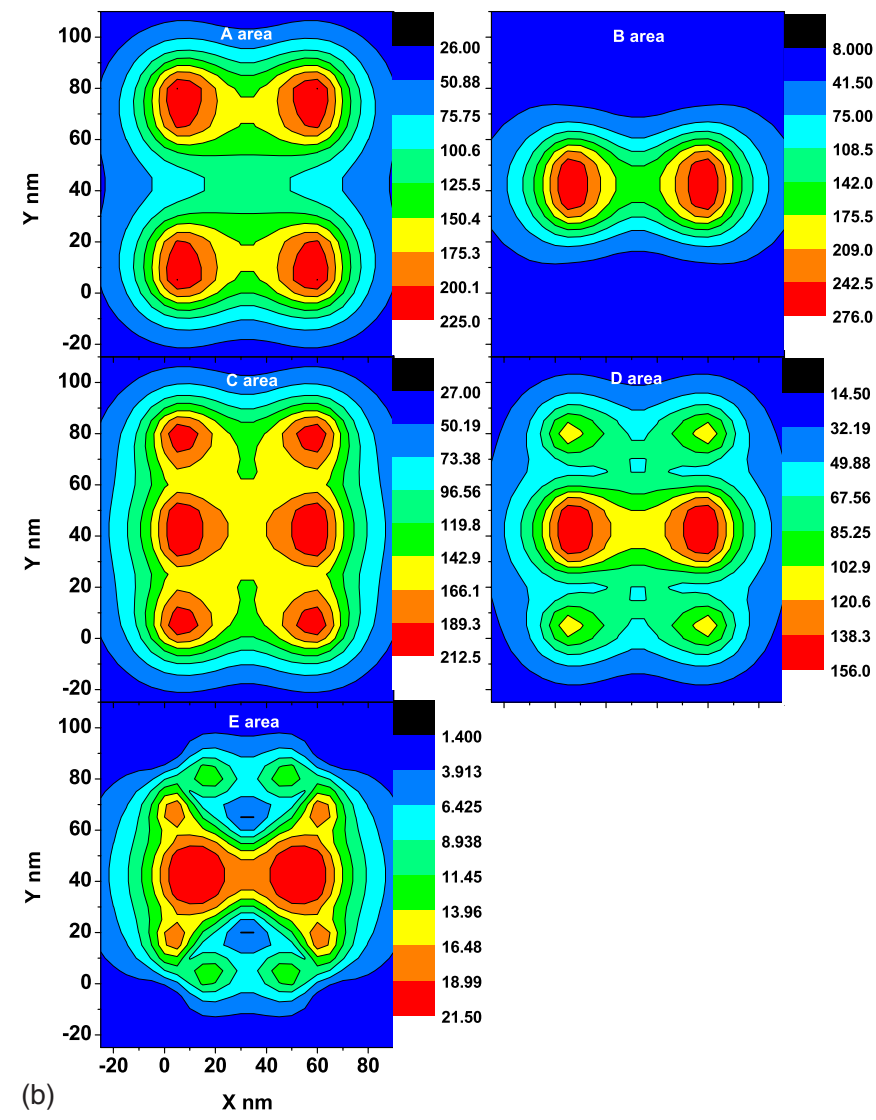

FIG. 5. (Color online) Near field distributions (a) within the nanostrips and (b) $10 \mathrm{~nm}$ above the nanostrips around the areas $A$, $B$, $C, D$, and $E$ in Fig. 4(b). 

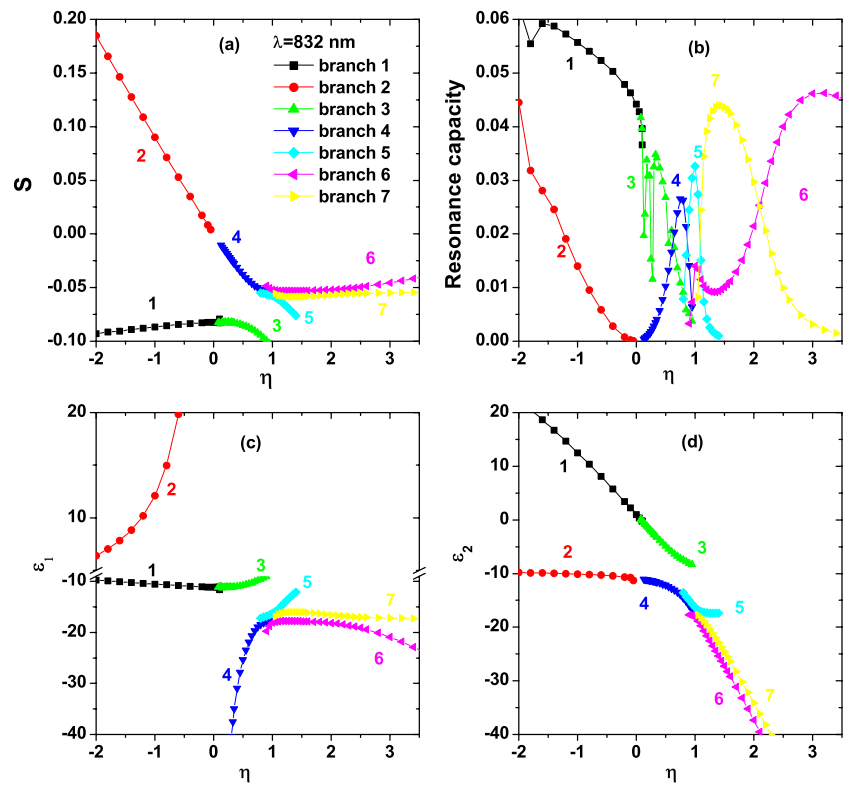

FIG. 6. (Color online) SPR with varying $\eta$ for the parallel case shown in Fig. 1(a) and at the wavelength $\lambda=832 \mathrm{~nm}$. (a) $\eta-s$ diagram, (b) $\eta-C_{n}$ diagram, (c) eigendielectric permittivity $\epsilon_{1}$, and (d) eigendielectric permittivity $\epsilon_{2}$. There are seven branches whose dielectric permittivities fall into the optical frequency range.

near field extending across all three-nanostrips is visible. As for branch 5 , resonances are dominated by $\epsilon_{2}$. In this branch $\epsilon_{1}$ changes very fast, but $\epsilon_{2}$ remains generally stable. Hence, if the $\epsilon_{1}$ material is $\mathrm{Au}$, its resonance wavelength will be very sensitive to the adjusting material $\epsilon_{2}$. From the above results, we find that in both the parallel and perpendicular cases, many resonance branches can be used to choose parameters which control the resonance wavelength and the resonance intensity. In the following, we will demonstrate this kind of control in the wavelength region of gold.

\section{C. $\eta$ - $s$ diagram at $\lambda=832 \mathrm{~nm}$}

We have provided the $\eta-s$ and $\eta-C_{n}$ (resonance capacity) diagrams of a three-strip nanosystem at $\lambda=632.8 \mathrm{~nm}$, namely, through the diagrams we can directly point out for a specific $\eta$ which dielectric permittivities $\epsilon_{1}$ and $\epsilon_{2}$ support the SPR. In practice, we need to map these resonances in the dielectric permittivity region into the resonance wavelength $\lambda_{R}$ region of real materials. It is easy to do this kind of mapping along the resonance branches in the $\eta-s$ diagram. According to the extension of $\epsilon_{1}(\in[-40,20])$ and $\epsilon_{2}(\in[$ $-40,20])$ at $\lambda=632.8 \mathrm{~nm}$, it is estimated that the resonances will cover the optical frequency region and will possess similar resonance characteristics over the wavelength range from $\lambda=500$ to $1000 \mathrm{~nm}$. In addition to the $\eta-s$ diagram at $\lambda$ $=632.8 \mathrm{~nm}$, we also provide the $\eta-s$ diagram at $\lambda$ $=832 \mathrm{~nm}$.

First, the parallel case of a three-strip nanostructure is presented. Comparing the $\eta-s$ diagrams at $\lambda=632.8 \mathrm{~nm}$ in Fig. 2 and at $\lambda=832 \mathrm{~nm}$ in Fig. 6 , it is found that they possess almost the same resonance properties, namely, there are seven main resonance branches and their resonance capacities and resonance $\epsilon_{1}$ and $\epsilon_{2}$ roughly fall into the same parameter regions. Next, we also compare the perpendicular
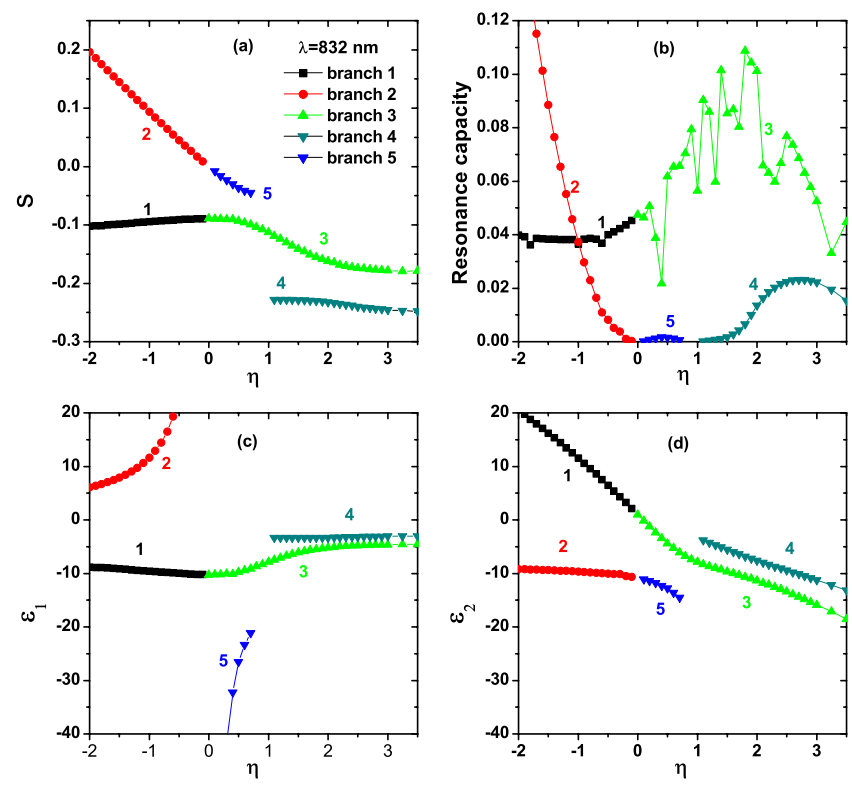

FIG. 7. (Color online) SPR with varying $\eta$ for the perpendicular case shown in Fig. 1(b) and at the wavelength $\lambda=832 \mathrm{~nm}$. (a) $\eta-s$ diagram, (b) $\eta$ $-C_{n}$ diagram, (c) eigendielectric permittivity $\epsilon_{1}$, and (d) eigendielectric permittivity $\epsilon_{2}$. There are five branches whose dielectric permittivities fall into the optical frequency range.

case at $\lambda=632.8 \mathrm{~nm}$ in Fig. 4 with that at $\lambda=832 \mathrm{~nm}$ in Fig. 7 , and almost the same results are found. Figures 6 and 7 illustrate these points in detail. This provides an important foundation to map resonances along their branches.

\section{CONTROLLING PLASMONIC PROPERTIES THROUGH BINARY NANOSTRUCTURES}

Plasmonic device design generally requires consideration of the resonance wavelength and the near field enhancement intensity. Resonances happen when the geometry of the nanostructure, the incident light, the metallic dielectric permittivity, and the dielectric environment match well. At present, there are two main ways to control the resonance properties. One is to fabricate different metallic nanoparticles, ${ }^{20}$ another is to rearrange the nanoparticles with different configurations and distances. ${ }^{10-13}$ Here, based on previous work on the interplay of plasmonic resonances in binary nanostructures, ${ }^{20}$ we propose using different material to control the plasmonic properties of the nanostructures. When one metallic material is used to control the SPR of another metal, it has a large effect on the resonance wavelength and the intensity due to the existence of free-electrons in the controlling nanostructure. In the following, for binary three-strip nanostructures, gold is used in two cases: (i) Au in the middle nanostrip and $\epsilon_{1}$ in the two outer strips, the results of which are shown in Figs. 9 and 11 and (ii) $\mathrm{Au}$ in the two outer nanostrips and $\epsilon_{2}$ in the middle strip, the results of which are shown in Figs. 8 and 10 . Then by adjusting $\epsilon_{1}$ in case (i) and $\epsilon_{2}$ in case (ii) we can control resonances. The size of the nanostrips is the same as that in Sec. III and the dielectric permittivity of $\mathrm{Au}$ is as given in Ref. 24. The labels of branches in Figs. 8 and 9 are the same as those in Fig. 2 and labels in Figs. 10 and 11 are as in Fig. 4. 


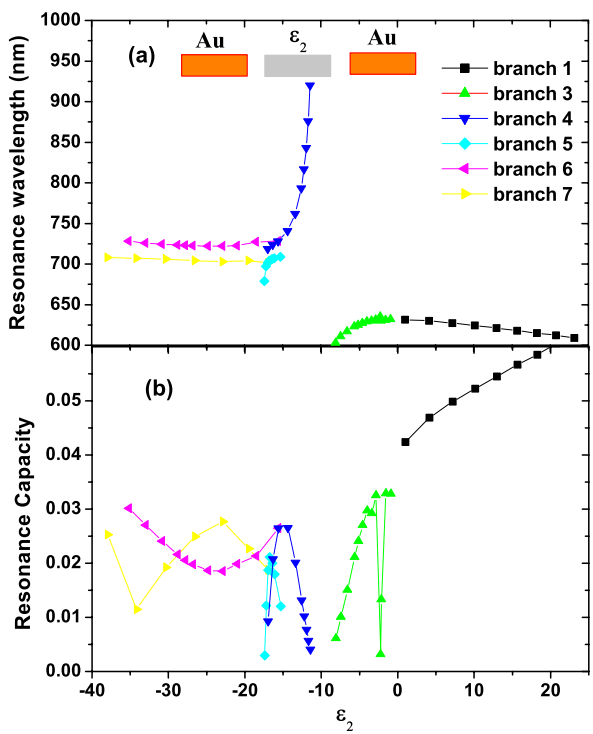

FIG. 8. (Color online) Plasmonic resonances of $\mathrm{Au}$ in a parallel binary three-strip nanostructure as a function of $\epsilon_{2}$ of the middle strip. (a) Resonance wavelength and (b) resonance capacity.

We first consider the situation where $\epsilon_{1}\left(\epsilon_{2}\right)>0$, while $\epsilon_{2}\left(\epsilon_{1}\right)>0$, namely, a dielectric material $\epsilon_{1}\left(\epsilon_{2}\right)$ is used to control the resonances of the metallic material $\epsilon_{2}\left(\epsilon_{1}\right)$. It is seen in branch 1 of Figs. 8 and 10 and in branch 2 of Figs. 9 and 11 that the resonance wavelength is nearly unchanged and the resonance capacity only has a very small modulation with variation in $\epsilon_{1}\left(\epsilon_{2}\right)$. Because the electromagnetic wave can penetrate through a dielectric, it is not suitable to adjust the plasmonic resonances.

Compared to $\epsilon_{\mathrm{Au}}(632.8 \mathrm{~nm})=-11.78$, too large $\epsilon_{1}$ (or $\epsilon_{2}$ ) or too small $\epsilon_{1}$ (or $\epsilon_{2}$ ) does not have much effect on the resonance wavelength, but they have a great effect on the resonance capacity. In the parallel case, as shown in Fig. 8 (or 9), when $\epsilon_{2}$ (or $\epsilon_{1}$ ) belongs to the region [-40,20], the resonance wavelengths are changed from $\lambda_{R}=700$ to $725 \mathrm{~nm}$

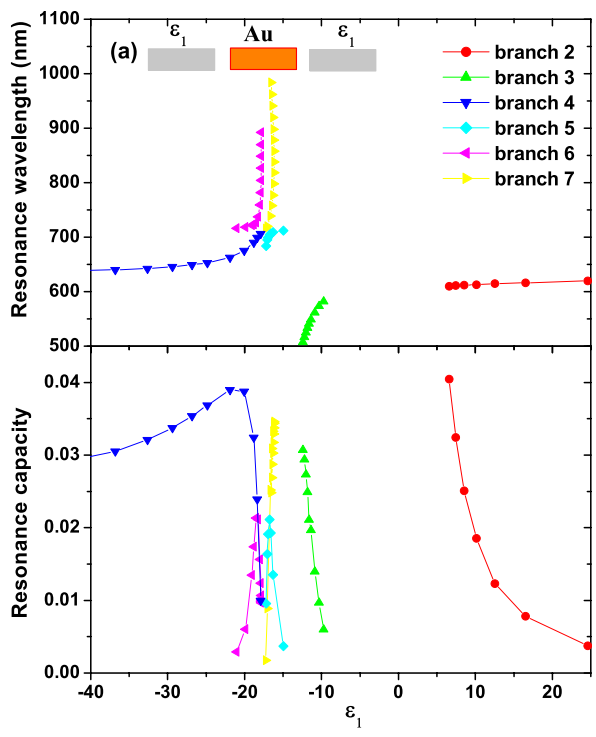

FIG. 9. (Color online) Plasmonic resonances of Au in a parallel binary three-strip nanostructure as a function of $\epsilon_{1}$ of both outer strips. (a) Resonance wavelength and (b) resonance capacity.

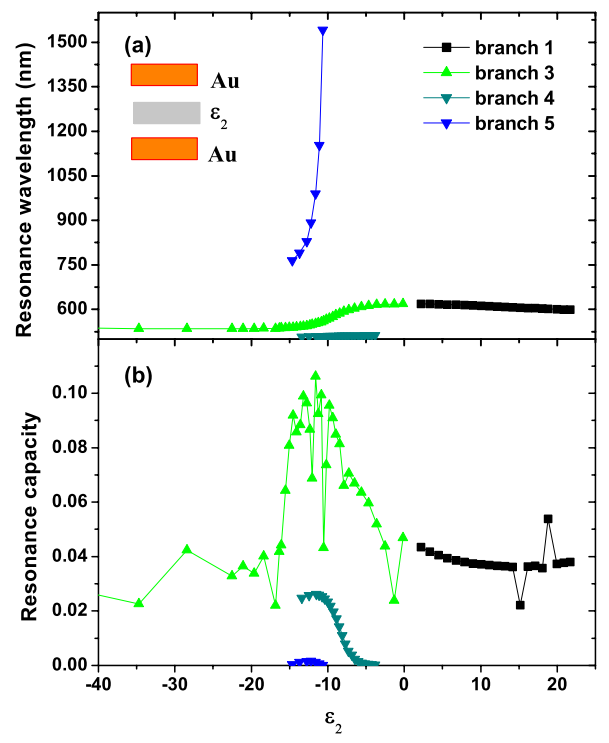

FIG. 10. (Color online) Plasmonic resonances of $\mathrm{Au}$ in a perpendicular binary three-strip nanostructure as a function of $\epsilon_{2}$ of the middle strip. (a) Resonance wavelength and (b) resonance capacity.

(or around $\lambda_{R}=650 \mathrm{~nm}$ ), and the resonance capacity can be modified greatly under the condition $\left|\epsilon_{2}\right|$ (or $\left.\left|\epsilon_{1}\right|\right)>\left|\epsilon_{\mathrm{Au}}\right|$, which corresponds to the $H$ (or $D$ ) area of Fig. 3. As for $\epsilon_{1}$ (or $\left.\epsilon_{2}\right) \in[-10,0]$, the resonance wavelength of $\mathrm{Au}$ and the resonance capacity have almost no visible changes, even in Fig. 9, we could not find strong resonances in this region.

Next, the perpendicular case is explored. As shown in Fig. 10 (or Fig. 11), almost the same things happen but with different parameter regions. When $\epsilon_{2}$ (or $\left.\epsilon_{1}\right) \in[-40,-15]$, the resonance wavelengths are changed around $\lambda_{R}$ $=630 \mathrm{~nm}$ (or from $\lambda_{R}=650$ to $675 \mathrm{~nm}$ ), and there is a comparable small resonance capacity under the condition $\left|\epsilon_{2}\right|$ (or $\left.\left|\epsilon_{1}\right|\right)>\left|\epsilon_{\mathrm{Au}}\right|$. Finally, for $\epsilon_{1}\left(\right.$ or $\left.\epsilon_{2}\right) \in[-5,0]$, the resonance wavelength and the capacity have almost no change and in Fig. 11 only many weak resonances exist (not shown

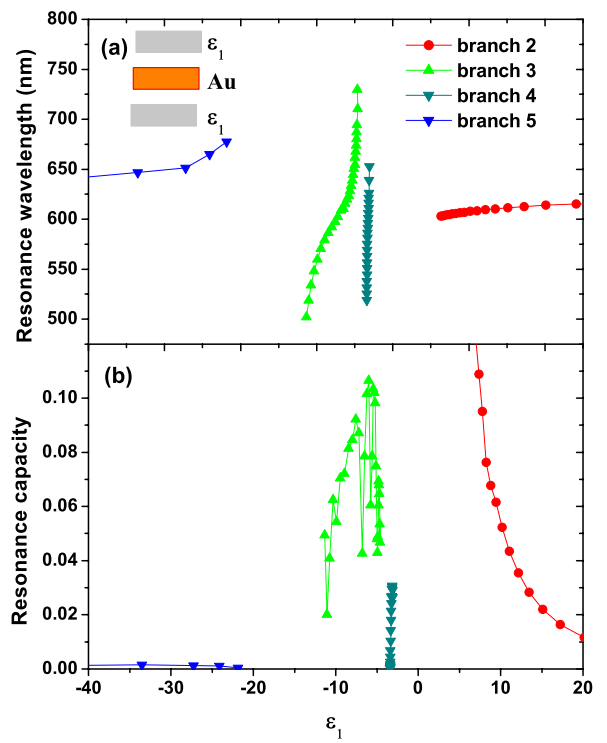

FIG. 11. (Color online) Plasmonic resonances of $\mathrm{Au}$ in a perpendicular binary three-strip nanostructure as a function of $\epsilon_{1}$ of both outer strips. (a) Resonance wavelength and (b) resonance capacity. 
here). However, why either the larger $\epsilon_{2}$ in branch 7 of Fig. 8 (in branch 3 of Fig. 10) or the larger $\epsilon_{1}$ in branch 4 of Fig. 9 (in branch 5 of Fig. 11) leads to the smaller resonance intensity is still an open question. A possible explanation may be related to the gathering and polarization of electrons. $^{23}$

The region where the permittivity parameter $\epsilon_{1}$ (or $\left.\epsilon_{2}\right)$ is comparable with $\epsilon_{\mathrm{Au}}(\omega)$ is discussed. In the parallel case as shown in Figs. 8 and 9, when $\epsilon_{1}$ (or $\left.\epsilon_{2}\right) \in[-20,-10]$, the resonance wavelength of $\mathrm{Au}$ can be tuned in a wide spectral region from $\lambda_{R}=650$ to $1000 \mathrm{~nm}$, and multibranch resonances coexist due to a strong interplay between two different materials. Moreover, in Fig. 8, at $\epsilon_{2}=-15$, there are three close resonance branches, belonging to branches 4,5 , and 6 , respectively. This multiresonance phenomenon should have some applications in plasmonic device design. ${ }^{25,26}$ For the perpendicular case, this region happens at about $\epsilon_{1}$ (or $\epsilon_{2}$ ) $\in[-15,-5]$, and almost the same features of resonance branches are found, namely, the resonance wavelength can be tuned from $\lambda_{R}=500$ to $1500 \mathrm{~nm}$, the resonance capacity has a large increment, and multibranch resonances coexist. It is noted in Fig. 10 that the resonance wavelength of $\mathrm{Au}$ can reach $\lambda_{R}=1500 \mathrm{~nm}$ near $\epsilon_{2}=-10$. So we conclude that for this kind of binary three-strip nanostructure the resonance wavelength and intensity can be well modulated at the region where the permittivity parameter $\epsilon_{1}$ (or $\epsilon_{2}$ ) can be competitive with the $\epsilon_{\mathrm{Au}}(\omega)$.

It is worth emphasizing the validity of the numerical results since the GMM does not include the loss of metals. This has been clarified in Ref. 20. From visible to nearinfrared, the dielectric permittivity $\left(\epsilon=\epsilon^{\prime}+i \epsilon^{\prime \prime}\right)$ of Au generally satisfies the condition $\left|\epsilon^{\prime}\right| \gg\left|\epsilon^{\prime \prime}\right|$, so, the imaginary part of dielectric permittivity will not have a noticeable influence on the resonance wavelength. The imaginary part $\epsilon^{\prime \prime}$ can, however, influence the width of the spectrum. In our previous work, ${ }^{19,27}$ a comparison between the GMM results and discrete dipole approximation ${ }^{28}$ results has been done. About 10-30 $\mathrm{nm}$ discrepancy in the resonance wavelength means a good agreement between both methods. So the results presented here are physically meaningful.

Finally, we use the Green's tensor technique to describe an example of plasmonic resonance control by means of $\mathrm{Au} / \mathrm{Ag}$ mixed metal. ${ }^{17}$ The results are also in agreement with the finite difference time domain (FDTD) and discrete dipole approximation (DDA) results. ${ }^{29}$ Using above nanostrip 60 $\times 20 \times 20 \mathrm{~nm}^{3}$ as a unit cell to show the absorption of $\mathrm{Au} /$ $\mathrm{Ag} / \mathrm{Au}$ and $\mathrm{Ag} / \mathrm{Au} / \mathrm{Ag}$ in the parallel and perpendicular cases, its resonance is at $\lambda_{R}=630 \mathrm{~nm}$ for $\mathrm{Au}$ and at $\lambda_{R}$ $=530 \mathrm{~nm}$ for Ag. However, when we mixed these unit cells together, for both $\mathrm{Au} / \mathrm{Ag} / \mathrm{Au}$ and $\mathrm{Ag} / \mathrm{Au} / \mathrm{Ag}$ parallel cases, their resonance wavelength is at $\lambda_{R}=725 \mathrm{~nm}$, as shown in Fig. 12(a). The dielectric permittivity $\epsilon_{\mathrm{Ag}}(725 \mathrm{~nm})$ is -25 . In Fig. 8, we observe a very good agreement, while, in Fig. 9 only a rough correspondence is found. For the perpendicular cases with $\mathrm{Au} / \mathrm{Ag} / \mathrm{Au}$ and $\mathrm{Ag} / \mathrm{Au} / \mathrm{Ag}$, their resonance wavelengths are $\lambda_{R}=550$ and $725 \mathrm{~nm}$ in Fig. 12(b). The dielectric permittivity $\epsilon_{\mathrm{Ag}}(550 \mathrm{~nm})$ is -13 . In Figs. 10 and 11, we have predicted this resonance at $\lambda_{R}=550 \mathrm{~nm}$. However, for the resonance at $\lambda_{R}=725 \mathrm{~nm}$, only a rough correspondence

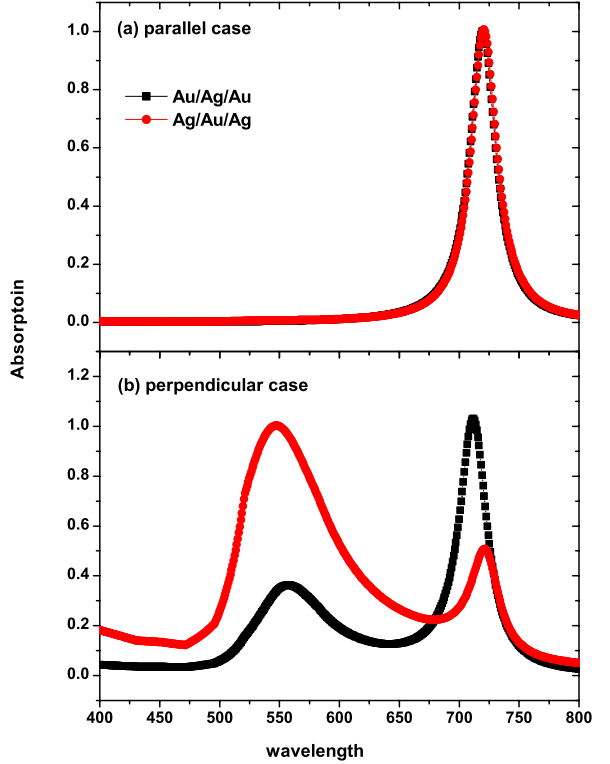

FIG. 12. (Color online) Normalized absorption cross sections of $\mathrm{Au} / \mathrm{Ag} / \mathrm{Au}$ and $\mathrm{Ag} / \mathrm{Au} / \mathrm{Ag}$ structures for the parallel and perpendicular arrangements.

is found in Fig. 11. We think that this discrepancy comes from the GMM, where we have not considered a loss in metal. However, $\eta-s$ and $\eta-C_{n}$ (resonance capacity) diagrams based on the GMM can give us a panorama of resonance behavior. For example, in the $\eta-s$ and $\eta-C_{n}$ diagrams, at $\lambda_{R}=550$ and $725 \mathrm{~nm}, \eta=1.83$ and $\eta=1.38$, which correspond to the region in which one material dominates. Hence, we conclude that in a hybrid nanostructure, SPR can be adjusted as well.

\section{SUMMARY}

In this work, using the recently developed GMM for solving SPR, we have investigated how to use material parameters in binary three-strip nanostructures to control plasmonic properties. We have first given their resonance branches for the parallel and perpendicular arrangement of three-nanostrips for fixed incident wavelengths $\lambda=632.8$ and $832 \mathrm{~nm}$, with varying the difference permittivity ratio $\eta$ $=\left(\epsilon_{2}-\epsilon_{0} / \epsilon_{1}-\epsilon_{0}\right)$. Enhanced near fields within the gaps or at the ends of the nanostrips can reach up to nine times that of a single nanostrip, due to the contribution of the freeelectrons in neighboring metallic materials. Then, we have mapped the resonances occurring in the permittivity region into the wavelength region for the real metal Au. By changing the dielectric permittivities $\epsilon_{1}$ or $\epsilon_{2}$, the resonance wavelength can be tuned from $\lambda_{R}=500$ to $1500 \mathrm{~nm}$, referred to a single nanostrip with $\lambda_{R}=630 \mathrm{~nm}$. Hence, through adjusting the material parameters, both resonance wavelength and resonance intensity can be controlled well. This binary threestrip nanostructure opens new possibilities for the design of hybrid plasmonic devices such as plasmonic sensors. 


\section{ACKNOWLEDGMENTS}

This work was supported by the National Natural Science Foundation of China under Grants Nos. 10874004, 10674009, and 10821062 and the National Key Basic Research Program (Grant No. 2007CB307001). Discussions with Professor Xiaoyong Hu are appreciated.

${ }^{1}$ A. Wokaun, J. P. Gordon, and P. F. Liao, Phys. Rev. Lett. 48, 957 (1982) ${ }^{2}$ S. Lal, S. Link, and N. J. Halas, Nat. Photonics 1, 641 (2007).

${ }^{3}$ P. Muhlschlegel, H. J. Eisler, O. J. F. Martin, B. Hecht, and D. W. Pohl, Science 308, 1607 (2005).

${ }^{4}$ J. R. Cole and N. J. Halas, Appl. Phys. Lett. 89, 153120 (2006).

${ }^{5}$ K. R. Catchpole and A. Polman, Opt. Express 16, 21793 (2008).

${ }^{6}$ A. Wokaun, J. G. Bergman, J. P. Heritage, A. M. Glass, P. F. Liao, and D. H. Olson, Phys. Rev. B 24, 849 (1981).

${ }^{7}$ E. M. Kim, S. S. Elovikov, T. V. Murzina, A. A. Nikulin, and O. A. Aktsipetrov, Phys. Rev. Lett. 95, 227402 (2005).

${ }^{8}$ M. Danckwerts and L. Novotny, Phys. Rev. Lett. 98, 026104 (2007).

${ }^{9}$ Y. Lu, G. L. Liu, J. Kim, Y. X. Mejia, and L. P. Lee, Nano Lett. 5, 119 (2005).

${ }^{10}$ H. Tamaru, H. Kuwata, H. T. Miyazaki, and K. Miyano, Appl. Phys. Lett. 80, 1826 (2002).

${ }^{11}$ L. Gunnarsson, T. Rindzevicius, J. Prikulis, B. Kasemo, M. Kall, S. Zou, and G. C. Schatz, J. Phys. Chem. B 109, 1079 (2005).
${ }^{12}$ P. K. Jain, S. Eustis, and M. A. El-Sayed, J. Phys. Chem. B 110, 18243 (2006).

${ }^{13}$ B. M. Reinhard, M. Siu, H. Agarwal, A. P. Alivisatos, and J. Liphardt, Nano Lett. 5, 2246 (2005).

${ }^{14}$ H. Xu, J. Aizpurua, M. Kall, and P. Apell, Phys. Rev. E 62, 4318 (2000).

${ }^{15}$ P. K. Jain, W. Y. Huang, and M. A. El-Sayed, Nano Lett. 7, 2080 (2007).

${ }^{16}$ P. K. Jain and M. A. El-Sayed, Nano Lett. 7, 2854 (2007).

${ }^{17}$ O. J. F. Martin, C. Girard, and A. Dereux, Phys. Rev. Lett. 74, 526 (1995).

${ }^{18}$ Y. Gu, K. W. Yu, and H. Sun, Phys. Rev. B 59, 12847 (1999).

${ }^{19}$ Y. Gu, L. L. Chen, H. X. Zhang, and Q. H. Gong, EPL 83, 27004 (2008).

${ }^{20}$ Y. Gu, Y. Wang, J. Li, O. J. F. Martin, and Q. H. Gong, Appl. Phys. B: Lasers Opt. 98, 353 (2010).

${ }^{21}$ P. M. Morse and H. Feshbach, Methods of Theoretical Physics (McGrawHill, New York, 1953).

${ }^{22}$ E. N. Economou, Green's Functions in Quantum Physics, 2nd ed. (Springer, Berlin, 1990).

${ }^{23}$ J. P. Kottmann, O. J. F. Martin, D. R. Smith, and S. Schultz, New J. Phys. 2, 27 (2000).

${ }^{24}$ P. B. Johnson and R. W. Christy, Phys. Rev. B 6, 4370 (1972).

${ }^{25}$ A. K. Sheridan, A. W. Clark, A. Glidle, J. M. Cooper, and D. R. S. Cumming, Appl. Phys. Lett. 90, 143105 (2007).

${ }^{26}$ A. W. Clark, A. K. Sheridan, A. Glidle, D. R. S. Cumming, and J. M. Cooper, Appl. Phys. Lett. 91, 093109 (2007).

${ }^{27}$ J. Li, Y. Gu, F. Zhou, Z. Y. Li, and Q. H. Gong, J. Mod. Opt. 56, 1396 (2009).

${ }^{28}$ P. K. Jain, K. S. Lee, I. H. El-Sayed, and M. A. El-Sayed, J. Phys. Chem. B 110, 7238 (2006).

${ }^{29}$ To avoid the mixing, the FDTD and DDA results are not shown here. 